Int. J. Morphol.,

26(4):823-824, 2008.

\title{
Erasistratus de Ceos (310-250 A.C.). Pionero de los Estudios Anatómicos
}

\author{
Erasistratus of Ceos (310-250 A.C.). Pioneer of the Anatomical Studies \\ Rafael Romero Reverón
}

ROMERO R. R. Erasistratus de Ceos (310-250 a.C.). Pionero de los estudios anatómicos. Int. J. Morphol., 26(4):823-824, 2008.

RESUMEN: Erasistratus de Ceos (310-250 a.C.) es considerado uno de los precursores del estudio e investigación en el área anatómica, realizando las primeras disecciones junto con Herophilus su maestro, fundador de las Escuelas de Medicina y Anatomía de Alejandría. Realizó importantes aportes en el estudio y enseñanza de la neuroanatomía, entre otras disciplinas anatómicas.

PALABRAS CLAVE: Erasistratus de Ceos; Historia de la Anatomía.

La historia de la Anatomía Humana como ciencia comienza con las inspecciones más tempranas de víctimas de sacrificios, pasando a la fundamental disección y posterior análisis más sofisticado del cuerpo humano realizado por modernos métodos tecnológicos, en la búsqueda de una comprensión continua de las funciones de órganos y de estructuras en el cuerpo humano.

Erasistratus de Ceos es considerado una de las primeras personas conocidas en la cultura occidental en estudiar e investigar sobre la anatomía humana a través de la disección anatómica durante el siglo III antes de Cristo. Sus estudios pueden considerarse como pioneros en la enseñanza y aprendizaje de la anatomía humana, por lo que me interese como Docente de la disciplina de Anatomía Normal, en investigar y recopilar la escasa información disponible para realizar esta síntesis de algunos aspectos de interés de la vida y obra de Erasistratus de Ceos, como parte de una área de investigación en desarrollo sobre personas que han realizado aportes significativos en la enseñanza y aprendizaje de esta rama del saber, como un conocimiento complementario en el estudio de la Anatomía Humana.

Erasistratus nació en Ceos en la Grecia antigua, proveniente de una familia médica. Su padre y hermano eran médicos y su madre era hermana de un médico. Él estudió Medicina en Atenas, alrededor 280 a.C., en el Centro de la Escuela Médica de Praxagoras.

Luego, Erasistratus se mudó a Alexandría, actual Egipto (en esos tiempos parte del mundo griego), en donde enseñó y practicó la medicina y fue médico real del rey Seleucus I de Siria. Contribuyó a fundar las antiguas y prestigiosas Escuelas de Anatomía y Medicina de Alejandría, así como el Museo de Alejandría, en el siglo III antes de Cristo (Puigbo, 2002; Eric Weisstein's World of Biography).
Erasistratus en conjunto con su maestro y contemporáneo Herophilus, fueron los primeras en hacer disecciones anatómicas en público y realizaron también, vivisecciones de criminales condenados.

Durante el breve período de tiempo en el historial médico griego de alrededor de 30-40 años, la prohibición para la disección humana fue levantada, Herophilus y Erasistratus tuvieron la oportunidad única de practicar la disección humana, una técnica de investigación no permitida, en ese tiempo, en ninguna otra parte del mundo, luego de lo cual esta práctica fue prohibida y no permitida otra vez, por 1800 años, hasta el siglo XVI después de Cristo, durante el Renacimiento (Sanabria, 1999; Erasistratus).

Erasistratus describió exactamente la estructura del cerebro, comparando los cerebros de seres humanos y de otros animales incluyendo las cavidades y las membranas, e hizo una distinción entre cerebro y cerebelo.

Él vio el cerebro y no al corazón, como hasta entonces se creía, la base de la inteligencia. Erasistratus de Ceos concluyó que un mayor número de giros del cerebro daba lugar a mayor inteligencia (Vargas, 1964; The Columbia Encyclopedia, 2001).

Él también describió exactamente la estructura y la función de los músculos gástricos y observó la diferencia entre los nervios motores y sensoriales (Vargas; The Columbia Encyclopedia). Erasistratus promovió la higiene, la dieta, y el ejercicio en la asistencia médica.

Erasistratus entendía que el corazón servía como una bomba, dilatando las arterias, describió y explicó el funcionamiento de las válvulas atrioventriculares derecha e izquierda. Él teorizó que tanto las arterias como venas, se extendían desde el 
corazón, dividiéndose finalmente en los tubos capilares extremadamente finos, que eran invisibles al ojo (Papp \& Agüero, 1994; Encyclopaedia Britannica).

Erasistratus de Ceos creía que las arterias estaban llenas de aire y que llevaban el "alcohol animal" del corazón. Esta sugerencia fue contra la creencia de entonces de los humores. Desarrolló una teoría reversa de la circulación. Se le atribuye a Erasistratus la primera descripción del ritmo cardiaco, después de curar a Nicanor, hijo del rey Selenucus I, midiéndole las palpitaciones del corazón, por lo que algunos lo consideran padre de la Fisiología (Viso, 1998; Wikipedia).

Estableció el nexo entre la afección mórbida en una víscera y su consecuencia clínica, mediante la observación y asociación entre el endurecimiento del hígado y la ascitis (Puigbo). Sin embargo, Erasistratus creía que el hígado formaba la sangre y la llevaba directo al corazón que, a su vez, lo bombeaba a los pulmones y de allí al resto de los órganos del cuerpo (Viso; Wikipedia). Erasistratus también creyó en un pneumapneuma, un alcohol animal vital, que fluye a través de los pulmones, al lado izquierdo del corazón y que este pneuma era bombeado a través de las arterias al resto del cuerpo. Los nervios, según Erasistratus, llevaban otra forma de pneuma.
En sus últimos años, Erasistratus se retiró de la práctica médica y contribuyó a ensamblar el Museo de Alexandría, en donde se dedicó a la investigación.

Aunque Erasistratus escribió extensivamente sobre un número de campos médicos, ninguno de sus trabajos sobreviven, todos sus escritos lamentablemente se perdieron en la destrucción de la biblioteca de Alexandría en el año 48 antes de Cristo (Allbiographies; Porter, 2004).

Después de Erasistratus de Ceos, la investigación anatómica con la disección terminó, debido a la presión de la opinión pública. Los egipcios creyeron en la necesidad de un cuerpo intacto para la vida futura eterna, por lo tanto, eran partidarios de la momificación y no de la disección.

Erasistratus en conjunto con su maestro y contemporáneo Herophilus, son los primeros anatomistas conocidos, al menos en la cultura occidental y quizás los primeros en realizar probablemente disecciones científicas de cuerpos humanos, por lo que son pioneros en el aprendizaje y enseñanza de la anatomía humana a través de la disecciones anatomícas. (Romero, 2007; Answer; University of Virginia)

ROMERO R. R. Erasistratus de Ceos (310-250 a.c.). Pionero de los estudios anatómicos. Int. J. Morphol., 26(4):823-824, 2008.

SUMMARY: Erasistratus of Ceos (310-250 b.C.) is considered one of the precursors of study and research in the anatomical area, doing the first dissections along with Herophilus, their teacher, founder of the Schools of Medicine and Anatomy of Alexandria. He made important contributions in the study and teaching of neuroanatomy, among other anatomical disciplines.

KEY WORDS: Erasistratus of Ceos; Anatomy's history.

\section{REFERENCIAS BIBLIOGRÁFICAS}

Allbiographies. Biography of Erasistratus. http:// www.allbiographies.com/biography- Erasistratus-11020.html

Answers. Erasistratus. /www.answers.com/topic/erasistratus?cat=health

Encyclopaedia Britannica. Erasistratus of Ceos (Greek physician) http:/ /www.britannica.com/eb/topic-191003/Erasistratus-of-Ceos

Erasistratus. www.in2greece.com/english/historymyth/history/ ancient/erasistratus.htm

Eric Weisstein's World of Biography. Erasistratus. http.// w w w. cienceworld.wolfram.com/biograph y/ Erasistratus.html

Papp, D. \& Agüero, A. Breve Historia de la Medicina. Editorial Claridad, Buenos Aires, Argentina, 1994. pp.81-2.

Porter, R. Breve Historia de la Medicina. México, Santillana Ediciones, 2004. p.98.

Puigbo, J. J. La Fragua de la Medicina Clinica y de la Cardiología. Universidad Central de Venezuela. Caracas, Consejo de Desarrollo Científico y Humanístico, 2002. pp.95-6.
Romero, R. Herófilo (335 - 280 a.C.): Pionero de la disección anatómica humana. Rev. de Federación Médica Venezolana, 15(1):33-4, 2007.

Sanabria, A. Compendio de Historia Universal de la Medicina y la Medicina Venezolana. Universidad Central de Venezuela. Caracas, Ediciones de la Biblioteca EBUC, 1999. p. 21.

The Columbia Encyclopedia, Erasistratus. $6^{\text {th }}$ Edition, 2001. www.bartleby.com/65/er/Erasistr.html

University of Virginia. Health System. Alexandrian Medicine www.mic.ki.se/West.html

Vargas, M. J. Obras Completas compiladas y notas por Dr. Blas Bruni Celli Anatomía. Tomo I. Caracas, Imprenta Nacional. 1964. pp. 40-1.

Viso, J. Nomenclatura Anatómica Moderna. Caracas, Universidad Central de Venezuela, 1998. p18.

Wikipedia. Erasistratus. http://en.wikipedia.org/wiki/Erasistratus.

Dirección para correspondencia:

Dr. Rafael Romero Reverón.

Cátedra de Anatomía Normal, Escuela de Medicina J.M. Vargas,

Facultad de Medicina, Universidad Central de Venezuela.

Email: romeroreveron@cantv.net rafa1636@yahoo.es

Recibido : 11-06-2008

Aceptado: 28-10-2008 